Osler Chest Unit, Oxford Radcliffe

Trust,

Headington, Oxford

OX3 7LJ, UK

C W H Davies

Chest Department,

Wycombe Hospital,

High Wycombe,

Buckinghamshire

HP11 2TT, UK

C I Mackinlay

C G Wathen

Correspondence to: Dr C W H Davies.

Received 12 August 1996

Returned to authors

8 January 1997

8 January 1997
Revised version received

Revised version r
21 January 1997

21 January 1997
Accepted for publication

Accepted for publicat
19 February 1997

\section{Recurrent post-partum pulmonary eosinophilia}

\author{
C W H Davies, C I Mackinlay, \\ C G Wathen
}

\begin{abstract}
In 1980 a 23 year old woman developed idiopathic eosinophilic pneumonia which was successfully treated with corticosteroids. She subsequently developed two identical relapses in the post-partum period.

(Thorax 1997;52:1095-1096)
\end{abstract}

Keywords: idiopathic eosinophilic pneumonia, postpartum.

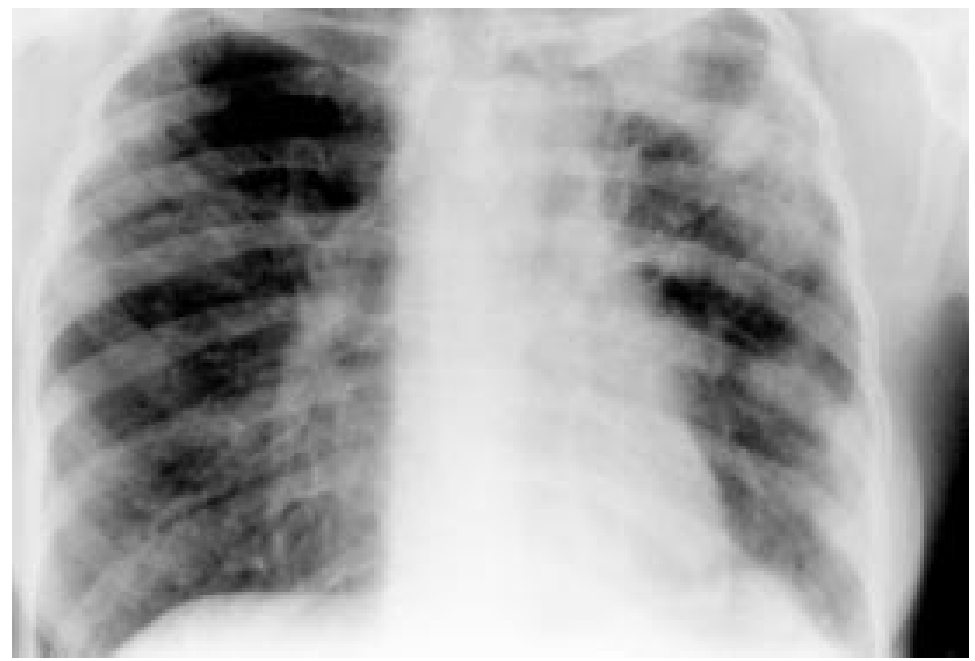

Figure 1 Chest radiograph in 1980 showing shadowing at the left lung apex.

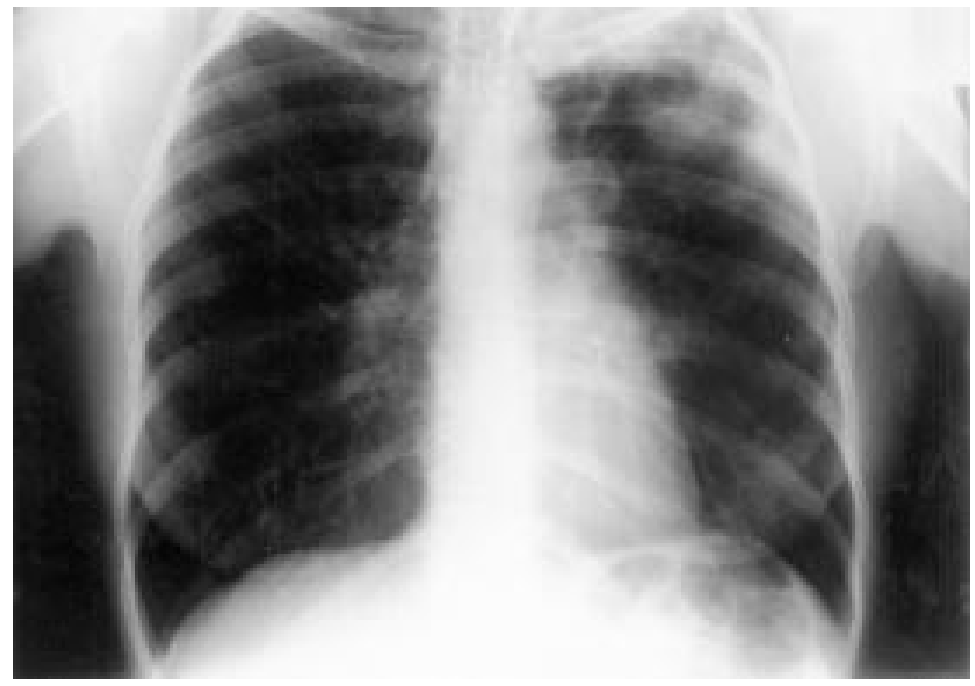

Figure 2 Chest radiograph at time of latest relapse in 1996 showing a similar appearance to fig 1 .
Chronic eosinophilic pneumonia is an intermittent pulmonary infiltrative syndrome with a tendency to relapse after discontinuation of steroid treatment. The aetiology and precipitants of such relapses are unknown. ${ }^{1}$ We describe a case of chronic eosinophilic pneumonia with relapse on two occasions in the puerperal period.

\section{Case report}

In 1981 a 23 year old female doctor with mild asthma became acutely unwell with a three week history of weight loss, night sweats, malaise and dyspnoea. She became unable to climb a flight of stairs and was admitted to her teaching hospital. There was no history of foreign travel. She was on the oral contraceptive pill but no other medications. Chest radiography showed airspace shadowing in the left lung apex (fig 1), suspected to be due to tuberculosis. However, the blood count showed leucocytosis with $50 \%$ eosinophilia. Bronchoalveolar lavage and transbronchial biopsy confirmed an eosinophilic infiltrate and culture was negative. Stool samples were negative for ova/parasites. Tuberculin and aspergillus skin prick testing were negative. A diagnosis of idiopathic eosinophilic pneumonia was made and she was commenced on prednisolone $60 \mathrm{mg}$ daily with an excellent response. She was able to discontinue treatment after eight months without immediate relapse.

Between 1982 and 1989 she remained well and required only inhaled salbutamol treatment for her asthma. In September 1989, six weeks post-partum, she became unwell again with recurrence of sweats, dyspnoea, weight loss, and no wheeze. She was not taking any medicines either during pregnancy or post-partum. Chest radiography showed similar airspace shadowing at the left apex and a blood count showed a markedly raised eosinophil count with a high erythrocyte sedimentation rate. She was again commenced on prednisolone with a rapid response to treatment. This was stopped after six months.

In 1992 she had an uneventful second pregnancy without relapse of pulmonary eosinophilia, but in January 1996 - six weeks postpartum - she developed identical symptoms to her previous relapses. There were no signs of systemic disease or vasculitis and she had not been on any medication. Again she had similar radiographic changes (fig 2), peripheral eosinophilia $\left(2.4 \times 10^{9} / 1 ; 23 \%\right.$ total white count) and raised erythrocyte sedimentation rate $(67 \mathrm{~mm} / \mathrm{h})$. Spirometric testing gave the following results: forced vital capacity $(\mathrm{FVC})=$ 3.2 litres ( $90 \%$ predicted), forced expiratory volume in one second $\left(\mathrm{FEV}_{1}\right)=2.2$ litres ( $70 \%$ predicted), $\mathrm{FEV}_{1} / \mathrm{FVC}=69 \%$ predicted Aspergillus precipitins, autoantibody screen, and anti-neutrophil cytoplasmic antibodies (ANCA) were negative. Urine microscopic ex- 
amination and culture was negative. The diagnosis of chronic eosinophilic pneumonia related to the post-partum period was made. She again made a full recovery on prednisolone and a repeat chest radiograph and computed tomographic (CT) scan of the thorax at this stage were normal, confirming resolution and excluding any anatomical abnormality. Her asthma is well controlled on inhaled budesonide and salbutamol.

\section{Discussion}

Chronic eosinophilic pneumonia was initially described by Carrington et al in 1969 as an idiopathic disease characterised by fever, dyspnoea, weight loss, and night sweats associated with diffuse infiltrates on chest radiography and pulmonary and peripheral eosinophilia. ${ }^{1}$ There is usually a dramatic response to corticosteroid treatment and a tendency to relapse after discontinuation. It has been reported in all adult age groups and both sexes but is more common in middle aged women. ${ }^{23}$ Some patients develop coexistent asthma, but this does not predict the clinical course. ${ }^{1}$ It is essential to exclude other recognisable causes and associations of peripheral eosinophilia and pulmonary infiltrates, especially allergic bronchopulmonary aspergillosis (ABPA), infections, drugs, parasites, systemic lupus erythematosus (SLE), sarcoidosis, and vasculitis. ${ }^{24}$

Our patient had confirmed idiopathic eosinophilic pneumonia in 1982 whilst taking the oral contraceptive. The diagnosis was based on peripheral and pulmonary eosinophilia, a rapid response to corticosteroids, and no identifiable cause after intensive investigation. She continued to suffer from controlled mild asthma but on two occasions developed typical features of chronic eosinophilic pneumonia during the post-partum period.

We have only found three cases in the literature relating to pregnancy and eosinophilic pneumonia. Tosini et al reported an uneventful pregnancy in a 24 year old woman with known chronic eosinophilic pneumonia whose respiratory symptoms were controlled by increased corticosteroids at the time of deterioration. ${ }^{5}$ Tohya et al described the onset of pulmonary infiltrates and peripheral eosinophilia in a 28 year old pregnant woman who was assumed to have eosinophilia secondary to antibiotics received previously. ${ }^{6}$ The patient made an initial recovery without treatment, but subsequently relapsed six months post-partum re- quiring corticosteroid therapy. The presence of pulmonary eosinophilia was not confirmed but she made a full recovery.

Dothager and Kollef reported typical symptoms of chronic eosinophilic pneumonia in a 22 year old woman during the sixth month of pregnancy. ${ }^{7}$ Her symptoms persisted into the post-partum period and the diagnosis was confirmed by bronchoalveolar lavage. She recovered with corticosteroids without relapse.

To our knowledge, recurrent post-partum eosinophilic pneumonia has not been reported. It is possible that the relationship between relapse and the puerperium is due to changes in glucocorticoids during pregnancy, as has been suggested for improvements in sarcoidosis during pregnancy. ${ }^{8}$ Maternal levels of cortisol and cortisol binding globulin increase during the second and third trimesters with an associated reduction in cortisol clearance and increased free cortisol levels. These changes are reversed post-partum. ${ }^{9}$ It may be that oestrogens can precipitate eosinophilia during pregnancy which is suppressed by glucocorticoids and then uncovered post-partum. These changes may have revealed the underlying chronic eosinophilic pneumonia at this phase in our patient. There is some evidence that the peripheral blood eosinophil count rises transiently one month post-partum in Japanese women. ${ }^{10}$ It is not clear why the second pregnancy was uneventful.

This is a unique case of chronic eosinophilic pneumonia. We believe that pregnancy and the puerperal period should be included in the list of recognised associations with this condition.

We would like to thank Dr M Green for his assistance with this case report.

1 Carrington CB, Addington WW, Goff AM, Madoff IM Marks A, Schwaber JR, et al. Chronic eosinophilic pneumonia. N Engl f Med 1969;280:787-98.

2 Allen JN, Davis WB. What is eosinophilic pneumonia? Arch Intern Med 1992;152:1765-6.

3 Douglas NJ, Goetzl EJ. In: Murray JF, Nadel JA, eds. Textbook of respiratory medicine. Philadelphia: W B Saunders, 1994: 1913-32.

4 Umeki S. Reevaluation of eosinophilic pneumonia and its diagnostic criteria. Arch Intern Med 1992;152:1913-9.

5 Tosini C, Faden D, Cattaneo R, Lojacono A, Tanzi P, Franzini $\mathrm{M}$, et al. Idiopathic eosinophilic pneumonia and pregnancy: report of a case. Int Arch Allergy Immunol 1995 106: $173-4$.

6 Tohya T, Matsui K, Itoh M, Okamura H. A case of pulmonary infiltration with eosinophilia syndrome in pregnancy. Acta Obstet Gynaecol fpn 1990;42:389-92.

nancy. Acta Obstet Gynaecol fpn 1990;42:389-92.
7 Dothager DW, Kollef MH. Postpartum pulmonary inDothager DW, Kollef MH. Postpartum pulmonary in-
filtrates with peripheral eosinophilia. Chest 1991;99:463-4. of a O. Sarcoidosis and pregnancy: a review with result of a retrospective survey. F Intern Med 1990;227:221-4. 9 Mastrogiannis DS, Whiteman VE, Mamopoulus M, Salameh WA. Acute endocrinopathies during pregnancy.
Clin Obstet Gynaecol 1994;37:78-92.

Nin Obstet Gynaecol 1994;37:78-92.

Miya Miyai $\mathrm{K}$. Changes of differential leukocyte counts during pregnancy and in the postpartum period. Rinsho Byor 